\title{
Hypothalamic loss of Snord116 and Prader-Willi syndrome hyperphagia: the buck stops here?
}

\author{
Juan A. Rodriguez ${ }^{1}$ and Jeffrey M. Zigman ${ }^{1,2,3}$ \\ 'Division of Hypothalamic Research, Department of Internal Medicine, ${ }^{2}$ Division of Endocrinology and Metabolism, Department of Internal Medicine, and ${ }^{3}$ Department of Psychiatry, University of Texas \\ Southwestern Medical Center, Dallas, Texas, USA.
}

\begin{abstract}
Hyperphagia and obesity are the best-known manifestations of PraderWilli syndrome (PWS) and are responsible for most of the overall morbidity and mortality associated with the disease. Yet these PWS symptoms remain poorly understood and without effective pharmacologic therapies. Mouse models attempting to recapitulate both the genetic alterations and marked hyperphagia plus obesity of PWS have been enigmatic, leading to skepticism about the use of mouse models to investigate PWS. In this issue of the $J C I$, Polex-Wolf and colleagues challenge the skeptics by successfully inducing hyperphagia following bilateral mediobasal hypothalamic deletion of the Snord116 gene from adult mice. Obesity also resulted, although only in a subset of mice. While this approach represents an exciting advance, highlighting a pathologic effect of loss of mediobasal hypothalamic Snord116 expression on the development of PWS's hallmark symptoms, the variability in the body-weight and body composition responses to this siteselective gene deletion raises several questions.
\end{abstract}

\section{Hyperphagia in Prader-Willi syndrome}

Prader-Willi syndrome (PWS) is a genetic disorder affecting multiple organ systems. Hypotonia is particularly prominent in the neonatal period, causing lethargy and a poor suck reflex, which in turn contribute to feeding difficulty and failure to thrive, often necessitating feeding-tube placement. Hypotonia in the respiratory musculature contributes to recurrent respiratory infections, which are another source of significant childhood morbidity and mortality (1-3). Individuals with PWS also often experience learning disabilities, disruptive behavioral problems, mental health issues, and a high pain threshold $(1,4,5)$. Furthermore, disorders of several endocrine systems are usually present, including, most prominently, growth-hormone deficiency $(1,6)$.

The preeminent symptom of PWS is hyperphagia, which develops at a median age of 8 years, is fueled by an insatiable appetite, and is manifested by a set of food-seeking behaviors that include binge eating, hoarding, pica, and food foraging, typically resulting in obesity despite careful monitoring (1, 6-9). This hyperphagia figures prominently in later childhood and into adulthood, contributing to most of the morbidity during that period and to early mortality, with most individuals with PWS not living past their early 40s (1). Earlier detection of the disorder, increased access for families to PWS resources and support groups, and the development of more inclusive, multidisciplinary treatment plans, which include growth-hormone replacement, have resulted in improvements in the overall management of PWS; however, the burden of disease stemming from hyperphagia remains high and without specific, highly efficacious therapeutic options pharmacologically based or otherwise.

Related Article: p. 960

Conflict of interest: The authors have declared that no conflict of interest exists

Reference information: J Clin Invest. 2018;128(3):900-902. https://doi.org/10.1172/JCI99725.

\section{Mouse models of PWS}

The creation of genetically modified mouse models targeting the same chromosomal regions affected in humans with PWS represents one approach to gaining a better understanding of the biological basis for the hyperphagia of PWS and for the development and testing of potential treatments. Over thirty genetic mouse models of PWS have been generated by deleting fragments of mouse chromosome 7 that correspond to segments of human chromosome 15 associated with PWS (10). The sporadic loss of or failure to express one or more of the paternally expressed genes within the human chromosome 15 segment results in PWS (1, 10). Unfortunately, mice lacking the entire PWS domain homolog die soon after birth (10). While several mouse models lacking individual genes within the PWS region have better survivability, they often manifest only a subset of the phenotypes that characterize PWS $(10,11)$.

One such single-gene deletion model of PWS is the Magel2-null mouse. This line carries a paternally inherited lacZ gene in place of the Magel2 coding sequence and thus lacks the PWS-implicated gene Magel2. Moreover, this model recapitulates several PWS-associated phenotypes, including hypotonia, increased adiposity, and hyperphagia. In particular, male and female Magel2-null mice exhibit increased fat mass by 18 weeks of age whether fed regular chow or high-fat diet (HFD) (12, 13). Although these animals have been characterized as obese, perhaps they would be more accurately characterized as having increased adiposity instead of obesity per se, as increases in body weight, when observed in females on HFD, remain slight $(12,13)$. Similarly to what occurs in PWS, Magel2-null mice show reduced energy expenditure, reduced lean mass in females, and decreased muscle tone and strength $(12,13)$. Importantly, both male and female Magel2-null mice exhibit hyperphagia when fed $\operatorname{HFD}(12,13)$. 
Another single-gene deletion model of PWS is the Snord116 $6^{+/-P}$ mouse, with a congenital paternal Snord116 deletion (14). Of note, a human subject with a microdeletion limited to the SNORD116 gene cluster displayed many similarities, including hyperphagia, excessive weight gain, and obesity beginning in early childhood, observed in PWS subjects with more common chromosomal deletions of the entire PWS critical region (15). Of the many key PWS features recapitulated within Snord116 $6^{+/ P}$ mice, growth delay marked by reduced body weight and reduced body length and an impaired growth-hormone pathway seem most prominent, while frank hypotonia is not present $(14,16,17)$. As regards to the eating and body-weight phenotype of adults with PWS, some studies have described Snord $116^{+/-P}$ mice as exhibiting slight hyperphagia, but only when their body weights are taken into consideration $(14,16,17)$. In other words, while the lighter Snord116 ${ }^{+/-P}$ mice have been shown to eat more food per body weight and demonstrate higher body-weight gain, individual Snord116 ${ }^{+/-P}$ mice do not eat more food than the heavier WT mice. Prolonged eating bouts, which suggest impaired satiation, and increased food intake during a fast-refeed protocol also have been noted in Snord116 ${ }^{+/-P}$ mice. That said, Snord $116^{+/-P}$ mice do not become obese (14), nor do mice with biallelic Snord116 deletion become obese (18, 19). Importantly, as opposed to what occurs in PWS, which is associated with reduced energy expenditure, adult Snord $116^{+/-P}$ mice and adult mice with biallelic Snord116 deletion display increased energy expenditure when housed at room temperature, which is speculated to block the development of obesity in the mice $(14,18,19)$, as likely does the initial growth delay.

\section{Hyperphagia plus obesity in a PWS mouse model}

The current work of Polex-Wolf and colleagues (20) moves to further investigate the role of Snord116 loss in the development of PWS. The authors took an existing floxed Snord116 mouse model $\left(\right.$ Snord116 $6^{f}$ ) in which loxP sites surround the Snord116 locus (14) and introduced adenoassociated virus (AAV) expressing Cre recombinase bilaterally into the mediobasal hypothalamus, thereby selectively deleting Snord116 expression with- in that region of the brain. Since these mice underwent the stereotaxic virus injections at 10 weeks of age, they developed without the prominent growth delay phenotype that characterizes Snor$d 116^{+/-P}$ mice. As compared with control mice receiving similar injections of AAV expressing GFP, mice correctly targeted with the Cre-expressing AAV exhibited hyperphagia when assessed 9 to 10 weeks after surgery using the analysis of covariance (ANCOVA) methodology, which corrects for body-weight differences. As an aside, Polex-Wolf and colleagues were unable to demonstrate hyperphagia in Snord116 ${ }^{+/-P}$ mice when the ANCOVA model was applied to the analysis. Notably, adult-onset mediobasal hypothalamic Snord116 deletion also resulted in obesity - as characterized by a body-weight gain over $140 \%$ of presurgery weight and the development of increased fat mass by 10 weeks after surgery - in five out of twentyone mice. No energy expenditure differences were noted between the Creexposed and GFP-exposed animals at six weeks after surgery, at which time bodyweight curves of the two groups began to diverge. Snord116 loss as a result of germline whole-body deletion in Snord116 $6^{+-P}$ mice or germline selective deletion from neuropeptide $\mathrm{Y}$ neurons, including orexigenic neuropeptide $\mathrm{Y}$ neurons in the mediobasal hypothalamus $(18,19)$, caused growth delay with lower body weights. On the other hand, the approach of AAVmediated mediobasal hypothalamic deletion of Snord116 from adult animals, which necessarily bypasses the neonatal period, resulted in both frank hyperphagia and, in a subset of animals, frank obesity.

\section{Questions raised}

Why did hyperphagia plus frank obesity develop only in five of the twenty-one mice? As a group, all twenty-one mice were hyperphagic, and the subset of five mice that gained over $140 \%$ of their presurgery weights and developed marked increases in fat mass were not any more hyperphagic than the sixteen other correctly targeted mice. Notably, higher expression of hypothalamic Socs 3 was detected in the obese subset. As Polex-Wolf et al. remark, Socs3 affects hypothalamic signaling pathways downstream of leptin and, when overexpressed in proopiomelanocortin (POMC) neurons, increases body weight and adiposity (21-23). Thus, further investigations into a potential connection between Snord116 and Socs 3 and a functional role of altered mediobasal hypothalamic Socs3 signaling in the development of hyperphagia and obesity associated with PWS seem warranted. Polex-Wolf and colleagues also discuss the possibility of polyphenism, in which disparate phenotypes occur despite underlying identical genotype and environmental conditions. Differential Snord116 loss is another potential factor in the variable expression of obesity. In particular, while the extents of mediobasal hypothalamic Cre expression achieved were similar in the two groups of mice, the investigators did not specifically determine whether there were any differences in Snord116 deletion between the subset that exhibited the more marked body-weight gain and the group that did not gain as much. A method that allows for a more uniform spatiotemporal targeting of Cre recombinase to the mediobasal hypothalamus or to specific mediobasal hypothalamic cell types would help reduce the potential for differential Snord116 deletion between mice in future studies. Although less likely, given that the gene manipulations were all done in adult Snord116 $6^{\mathrm{fl}}$ mice, it might also be worthwhile to determine whether differences in original litter size, genotypic or sex makeup of littermates, or maternal nurturing may have influenced the body-weight phenotypes of the mice. Additionally, it would be of interest to examine the effect of viralmediated mediobasal hypothalamic deletion of Snord116 from adult female mice, as the Polex-Wolf et al. study focused on males and as previous work with Magel2-null mice revealed higher body weights in female mice $(12,13)$.

Notably, a related approach, involving adult-onset whole-body Snord116 deletion, did not cause hyperphagia or obesity (24). Instead, these mice, which carried two Snord116 ${ }^{\text {fl }}$ genes and a gene expressing a tamoxifen-inducible Cre recombinase under the control of the ubiquitin promoter, and in which the Cre-mediated Snord116 deletion was induced at eight weeks of age, did not exhibit body-weight or body composition phenotypes and unexpectedly exhibited decreased food intake (24). While the expected body-weight and food-intake changes may not have been 
observed due to the partial (16\%) reduction in hypothalamic expression of Snord116 (24), one must also consider the possibility that differential loss of Snord116 in other tissues may somehow counter the effects of its loss in the mediobasal hypothalamus.

Additionally, Polex-Wolf and colleagues did not detect differences in hypothalamic expression of Pcsk1, which encodes prohormone convertase $1 / 3$, or Nhlh2, a transcription factor that regulates Pcsk1, in Snord116 ${ }^{+/-P}$ mice or in mice with viral-mediated mediobasal hypothalamic Snord116 deletion. This is seemingly counter to the recent JCI study by Burnett and colleagues in which iPS cell-derived (iPSC-derived) neurons from human subjects with the most common genetic form of PWS or with a microdeletion of the SNORD116 gene and two flanking genes were shown to display markedly downregulated expression of PCSK1 and NHLH2 (17). Reduced Nhlh2 and/or Pcsk1 expression also were observed in a number of tissues from Snord116 ${ }^{+/-P}$ mice, including the hypothalamus (17). Downregulation of these genes is predicted to restrict maturation of multiple hormones and neuropeptides, including several known to affect eating, body weight, and growth, throughout the body. Impaired prohormone processing of progrowth-hormonereleasing hormone, proinsulin, and proghrelin were all observed, and it was postulated that insufficient processing of mediobasal hypothalamic POMC or hypothalamic prooxytocin could contribute to the hyperphagia and obesity of PWS (17). Further exploration of the potential role of altered Pcsk1 expression, including the contribution of timing, in the phenotypic manifestations of PWS is warranted, especially given the lack of changes in Pcsk1 and Nhlh2 expression in Polex-Wolf et al.

\section{Final thoughts}

Polex-Wolf and colleagues have generated a new preclinical model of PWS, with both hyperphagia and obesity as prominent phenotypes, at least in a subset of individuals. While the apparent unpredictability in obesity occurrence might limit the utility of this model for testing compounds to prevent obesity in PWS, the model may be useful to test the anorexigenic actions of potential drugs and, in the subset of mice that do develop obesity, their weight-reducing efficacy.

\section{Acknowledgments}

This work was supported by the Foundation for Prader-Willi Research.

Address correspondence to: Jeffrey M. Zigman, University of Texas Southwestern Medical Center, 5323 Harry Hines Blvd., Dallas, Texas 75390-9077, USA. Phone: 214.648.8621; Email: jeffrey.zigman@ utsouthwestern.edu.

1. Cassidy SB, Schwartz S, Miller JL, Driscoll DJ. Prader-Willi syndrome. Genet Med. 2012;14(1):10-26.

2. de Lind van Wijngaarden RF, de Klerk LW, Festen DA, Duivenvoorden HJ, Otten BJ, Hokken-Koelega AC. Randomized controlled trial to investigate the effects of growth hormone treatment on scoliosis in children with Prader-Willi syndrome. J Clin Endocrinol Metab. 2009;94(4):1274-1280.

3. Shim JS, Lee SH, Seo SW, Koo KH, Jin DK. The musculoskeletal manifestations of Prader-Willi syndrome. J Pediatr Orthop. 2010;30(4):390-395.

4. Sinnema M, et al. Psychiatric illness in a cohort of adults with Prader-Willi syndrome. Res Dev Disabil. 2011;32(5):1729-1735

5. Skokauskas N, Sweeny E, Meehan J, Gallagher L. Mental health problems in children with Prader-Willi syndrome. JCan Acad Child Adolesc Psychiatry. 2012;21(3):194-203.

6. Miller JL. Approach to the child with PraderWilli syndrome. J Clin Endocrinol Metab. 2012;97(11):3837-3844.

7. Goldstone AP. Prader-Willi syndrome: advances in genetics, pathophysiology and treatment. Trends Endocrinol Metab. 2004;15(1):12-20.

8. Holm VA, et al. Prader-Willi syndrome: consensus diagnostic criteria. Pediatrics. 1993;91(2):398-402.

9. Miller JL, et al. Nutritional phases in PraderWilli syndrome. Am JMed Genet A. 2011;155A(5):1040-1049.

10. Resnick JL, Nicholls RD, Wevrick R, Prader-Willi
Syndrome Animal Models Working Group. Recommendations for the investigation of animal models of Prader-Willi syndrome. Mamm Genome. 2013;24(5-6):165-178.

11. Bervini S, Herzog H. Mouse models of PraderWilli Syndrome: a systematic review. Front Neuroendocrinol. 2013;34(2):107-119.

12. Knani I, et al. Targeting the endocannabinoid/CB1 receptor system for treating obesity in PraderWilli syndrome. Mol Metab. 2016;5(12):1187-1199.

13. Kamaludin AA, et al. Muscle dysfunction caused by loss of Magel2 in a mouse model of PraderWilli and Schaaf-Yang syndromes. Hum Mol Genet. 2016;25(17):3798-3809.

14. Ding F, et al. SnoRNA Snord116 (Pwcr1/MBII85) deletion causes growth deficiency and hyperphagia in mice. PLoS One. 2008;3(3):e1709.

15. Bieth $\mathrm{E}$, et al. Highly restricted deletion of the SNORD116 region is implicated in Prader-Willi Syndrome. Eur J Hum Genet. 2015;23(2):252-255.

16. Lin D, et al. Abnormal response to the anorexic effect of GHS-R inhibitors and exenatide in male Snord116 deletion mouse model for Prader-Willi syndrome. Endocrinology. 2014;155(7):2355-2362.

17. Burnett LC, et al. Deficiency in prohormone convertase PC1 impairs prohormone processing in Prader-Willi syndrome. J Clin Invest. 2017;127(1):293-305.

18. Qi Y, et al. Snord116 is critical in the regulation of food intake and body weight. Sci Rep. 2016;6:18614.

19. Qi Y, et al. Ambient temperature modulates the effects of the Prader-Willi syndrome candidate gene Snord116 on energy homeostasis. Neuropeptides. 2017;61:87-93.

20. Polex-Wolf J, et al. Hypothalamic loss of Snord116 recapitulates the hyperphagia of Prader-Willi syndrome. J Clin Invest. 2018;128(3):960-969.

21. Wunderlich CM, Hövelmeyer N, Wunderlich FT. Mechanisms of chronic JAK-STAT3-SOCS3 signaling in obesity. JAKSTAT. 2013;2(2):e23878.

22. Münzberg H, Myers MG. Molecular and anatomical determinants of central leptin resistance. Nat Neurosci. 2005;8(5):566-570.

23. Reed AS, Unger EK, Olofsson LE, Piper ML, Myers MG Jr, Xu AW. Functional role of suppressor of cytokine signaling 3 upregulation in hypothalamic leptin resistance and long-term energy homeostasis. Diabetes. 2010;59(4):894-906.

24. Purtell L, Qi Y, Campbell L, Sainsbury A, Herzog H. Adult-onset deletion of the Prader-Willi syndrome susceptibility gene Snord116 in mice results in reduced feeding and increased fat mass. Transl Pediatr. 2017;6(2):88-97. 